\title{
GENERICALLY FINITE MORPHISMS AND FORMAL NEIGHBORHOODS OF ARCS
}

\author{
LAWRENCE EIN AND MIRCEA MUSTAŢĂ
}

\begin{abstract}
Let $f: X \rightarrow Y$ be a morphism of pure-dimensional schemes of the same dimension, with $X$ smooth. We prove that if $\gamma \in J_{\infty}(X)$ is an $\operatorname{arc}$ on $X$ having finite order $e$ along the ramification subscheme $R_{f}$ of $X$, and if its image $\delta=f_{\infty}(\gamma)$ on $Y$ does not lie in $J_{\infty}\left(Y_{\text {sing }}\right)$, then the induced map $T_{\gamma} J_{\infty}(X) \rightarrow T_{\delta} J_{\infty}(Y)$ is injective, with a cokernel of dimension $e$. In particular, if $Y$ is smooth too, and if we denote by $\widehat{J_{\infty}(X)_{\gamma}}$ and $\widehat{J_{\infty}(Y)_{\delta}}$ the formal neighborhoods of $\gamma \in J_{\infty}(X)$ and $\delta \in J_{\infty}(Y)$, then the induced morphism $\widehat{J_{\infty}(X)_{\gamma}} \rightarrow \widehat{J_{\infty}(Y)} \delta$ is a closed embedding of codimension $e$.
\end{abstract}

\section{IntroduCtion}

All schemes and all scheme morphisms are defined over an algebraically closed field $k$ of arbitrary characteristic. $X$ and $Y$ denote schemes of finite type over $k$. We are interested in local questions, hence we may and will assume that $X$ and $Y$ are affine.

The scheme of arcs of $X$ is denoted by $J_{\infty}(X)$. For every $k$-algebra $A$, we have a natural bijection

$$
\operatorname{Hom}\left(\operatorname{Spec} A, J_{\infty}(X)\right) \simeq \operatorname{Hom}(\operatorname{Spec} A \llbracket t \rrbracket, X) .
$$

In particular, a $k$-valued point of $J_{\infty}(X)$ corresponds to an arc $\gamma$ : Spec $k \llbracket t \rrbracket \rightarrow X$ (we stress that all arcs that we consider are $k$-valued arcs). A morphism $f: X \rightarrow Y$ induces a morphism between the schemes of $\operatorname{arcs} f_{\infty}: J_{\infty}(X) \rightarrow J_{\infty}(Y)$. If $\gamma$ is an $\operatorname{arc}$ in $X$, and if $Z$ is a closed subscheme of $X$ defined by the ideal $I_{Z}$, then the order of vanishing $\operatorname{ord}_{Z}(\gamma)$ is that $e \in \mathbf{Z}_{\geq 0} \cup\{\infty\}$ such that the inverse image $\gamma^{-1} I_{Z} \subseteq k \llbracket t \rrbracket$ is equal to ( $\left.t^{e}\right)$ (with the convention that $e=\infty$ if this ideal is zero). For an introduction to spaces of arcs and some applications to the study of singularities of pairs, see for example [EM].

If $f: X \rightarrow Y$ is a morphism of schemes of pure dimension $n$, then we denote by $R_{f}$ the ramification subscheme of $X$, defined by $\operatorname{Fitt}^{0}\left(\Omega_{X / Y}\right)$, the $0^{\text {th }}$ Fitting ideal of the sheaf of relative differentials $\Omega_{X / Y}$. If $X$ and $Y$ are integral schemes, and $f$ is dominant, then $f$ is separable if and only if $R_{f}$ contains no component of $X$. Note also that if both $X$ and $Y$ are smooth and irreducible, and $f$ is dominant, then $R_{f}$ is the

The first author was partially supported by NSF grant DMS-0700774, and the second author was partially supported by NSF grant DMS-0758454 and by a Packard Fellowship. 
degeneracy locus of the morphism $f^{*} \Omega_{Y}^{1} \rightarrow \Omega_{X}^{1}$ of locally free sheaves of rank $n$. In particular, in this case $R_{f}$ is either a divisor, or $R_{f}=X$ (when $f$ is inseparable).

If $\gamma$ is a $k$-valued point of $J_{\infty}(X)$, then we denote by $\widehat{J_{\infty}(X)_{\gamma}}$ the formal neighborhood of $\gamma$ in $J_{\infty}(X)$, that is, the formal completion of $J_{\infty}(X)$ at the point $\gamma$. We put $\widehat{\mathbf{A}_{0}^{n}}$ for the formal neighborhood of the origin in $\mathbf{A}^{n}$. Note that for every arc $\gamma$ and every $e$, the closed immersion $J_{\infty}(X) \hookrightarrow J_{\infty}(X) \times \mathbf{A}^{e}$ given by $\alpha \rightarrow(\alpha, 0)$ induces by passing to formal neighborhoods a closed immersion $\iota_{\gamma, e}: \widehat{J_{\infty}(X)_{\gamma}} \hookrightarrow \widehat{J_{\infty}(X)_{\gamma}} \widehat{\times} \widehat{\mathbf{A}_{0}^{e}}$. The following is our main result.

Theorem 1.1. Let $f: X \rightarrow Y$ be a morphism of smooth varieties of pure dimension $n$. If $\gamma$ is an arc on $X$ with $\operatorname{ord}_{R_{f}}(\gamma)=e<\infty$, and if $\delta=f_{\infty}(\gamma)$, then we have an isomorphism

$$
\widehat{J_{\infty}(Y)_{\delta}} \simeq \widehat{J_{\infty}(X)_{\gamma}} \widehat{\times} \widehat{\mathbf{A}_{0}^{e}}
$$

such that the morphism induced by $f$ corresponds to $\iota_{\gamma, e}$.

It is interesting to compare this statement with the geometric version of the Change of Variable formula for motivic integration, due to Kontsevich [Kon] and Denef and Loeser [DL]. That result says that if $f$ is birational and if we consider instead the finite-level truncation morphisms $f_{m}: J_{m}(X) \rightarrow J_{m}(Y)$, with $m \geq 2 e$, then on the locus where the order of vanishing along $R_{f}$ is $e$, the map $f_{m}$ is piecewise trivial, with fiber $\mathbf{A}^{e}$. In fact, the key argument in the proof of Theorem 1.1 is similar to the one in $[\mathrm{DL}]$.

We will deduce Theorem 1.1 from a statement involving only the tangent spaces of the corresponding spaces of arcs. Note that in this case we do not need $Y$ to be smooth.

Theorem 1.2. Let $f: X \rightarrow Y$ be a morphism of pure-dimensional schemes of the same dimension, with $X$ smooth. If $\gamma$ is an arc in $X$ with $\operatorname{ord}_{R_{f}}(\gamma)=e<\infty$, and if $\delta:=f_{\infty}(\gamma) \notin J_{\infty}\left(Y_{\text {sing }}\right)$, then the induced tangent map

$$
T_{\gamma} J_{\infty}(X) \rightarrow T_{\delta} J_{\infty}(Y)
$$

is injective, and its cokernel has dimension e.

We mention that if $X$ is a scheme of finite type over $k$, then a theorem of Grinberg and Kazhdan [GK] describes the formal neighborhood of a $k$-valued arc $\gamma \in J_{\infty}(X) \backslash$ $J_{\infty}\left(X_{\text {sing }}\right)$ as the product of the formal neighborhood of some point on a scheme of finite type, with the formal neighborhood of the origin on an infinite dimensional affine space. This result was reproved by Drinfeld in [Dr] using a projection of $X$ to an affine space. It would be interesting to extend the result in Theorem 1.2 to get more information on $\widehat{J_{\infty}(Y)_{\delta}}$, and compare this approach to the one in loc. cit. 
Our interest in Theorem 1.1 is motivated by possible applications to the study of singularities. The Change of Variable formula in [DL] can be applied to describe invariants of singularities of pairs in terms of codimensions of contact loci in spaces of arcs in the presence of a resolution of singularities (see [EM]). In positive characteristic however, resolutions are not available, the only possible tool at this point being de Jong's alterations [deJ]. The Change of Variable formula being restricted to birational morphisms, we view Theorem 1.1 as a possible replacement in this setting. We hope to return to this problem in the future.

\section{The tangent MAP OF $f_{\infty}$}

In this section we prove Theorem 1.2 above. As we have mentioned, the argument is a variation on the argument in Lemma 3.4 in [DL] (see also [Lo] and [EM]). We start by recalling some basic facts about Fitting ideals that we will use (see [Eis] for details and proofs).

Suppose that $\mathcal{F}$ is a coherent sheaf on a Noetherian scheme $W$. If $\mathcal{F}$ has a free presentation

$$
\mathcal{O}_{W}^{\oplus a} \stackrel{\varphi}{\rightarrow} \mathcal{O}_{W}^{\oplus b} \rightarrow \mathcal{F} \rightarrow 0
$$

then $\operatorname{Fitt}^{r}(\mathcal{F})$ is the ideal generated by the $(b-r)$-minors of $\varphi$ (by convention, this is $\mathcal{O}_{W}$ if $\left.r \geq b\right)$. In general, Fitting ideals are compatible with pull-back: if $h: W^{\prime} \rightarrow W$ is a morphism of schemes, then $\operatorname{Fitt}^{r}\left(h^{*} \mathcal{F}\right)=\operatorname{Fitt}^{r}(\mathcal{F}) \cdot \mathcal{O}_{W^{\prime}}$. Since we work over a perfect base field, if $Y$ is a scheme of finite type over $k$, of pure dimension $n$, then the singular locus of $Y$ is defined by $\operatorname{Fitt}^{n}\left(\Omega_{Y}\right)$.

Suppose now that $M$ is a finitely generated module over $k \llbracket t \rrbracket$. Then

$$
M \simeq k \llbracket t \rrbracket^{\oplus m} \oplus k[t] /\left(t^{q_{1}}\right) \oplus \cdots \oplus k[t] /\left(t^{q_{s}}\right)
$$

for some $m, s \geq 0$ and $q_{1}, \ldots, q_{s} \geq 1$. In this case $m=\operatorname{dim}_{k((t))} M \otimes_{k \llbracket t \rrbracket} k((t))$, and $\operatorname{Fitt}^{m}(M)=\left(t^{\sum_{i} q_{i}}\right)$.

Proof of Theorem 1.2. Let $\gamma \in J_{\infty}(X)$ be an arc lying over $x \in X$, that we identify with the corresponding homomorphism $\mathcal{O}_{X, x} \rightarrow k \llbracket t \rrbracket$. A tangent vector at $\gamma$ to $J_{\infty}(X)$ corresponds to a homomorphism $\widetilde{\gamma}: \mathcal{O}_{X, x} \rightarrow k \llbracket s, t \rrbracket /\left(s^{2}\right)$ of the form $\gamma+s D$ for some $D: \mathcal{O}_{X, x} \rightarrow k \llbracket t \rrbracket$. The condition that $\widetilde{\gamma}$ is a homomorphism is equivalent to $D$ being a derivation, where $k \llbracket t \rrbracket$ is an $\mathcal{O}_{X, x}$-module via $\gamma$. In other words,

$$
T_{\gamma} J_{\infty}(X) \simeq \operatorname{Hom}_{k \llbracket t \rrbracket}\left(\Omega_{X, x} \otimes_{\mathcal{O}_{X, x}} k \llbracket t \rrbracket, k \llbracket t \rrbracket\right) .
$$

A similar description holds for $T_{\delta} J_{\infty}(Y)$, where $\delta=f_{\infty}(\gamma)$.

Let $y=f(x)$. If we pull-back via $\gamma$ the exact sequence

$$
f^{*} \Omega_{Y} \rightarrow \Omega_{X} \rightarrow \Omega_{X / Y} \rightarrow 0,
$$


we get an exact sequence

$$
\Omega_{Y, y} \otimes_{\mathcal{O}_{Y, y}} k \llbracket t \rrbracket \stackrel{A}{\rightarrow} \Omega_{X, x} \otimes_{\mathcal{O}_{X, x}} k \llbracket t \rrbracket \rightarrow \Omega_{X / Y} \otimes_{\mathcal{O}_{X, x}} k \llbracket t \rrbracket \rightarrow 0 .
$$

By hypothesis $\delta \notin J_{\infty}\left(Y_{\text {sing }}\right)$, hence $\delta$ maps the generic point of Spec $k \llbracket t \rrbracket$ to the nonsingular locus of $Y$. Let $n=\operatorname{dim}(X)=\operatorname{dim}(Y)$. Since the ground field is perfect, we deduce $\operatorname{dim}_{k((t))}\left(\Omega_{Y, y} \otimes k((t))\right)=n$. Therefore

$$
\Omega_{Y, y} \otimes k \llbracket t \rrbracket \simeq k \llbracket t \rrbracket^{\oplus n} \oplus k[t] /\left(t^{q_{1}}\right) \oplus \cdots \oplus k[t] /\left(t^{q_{s}}\right),
$$

for some $q_{1}, \ldots, q_{s} \geq 1$. Moreover, $X$ is smooth, hence $\Omega_{X, x} \otimes k \llbracket t \rrbracket \simeq k \llbracket t \rrbracket^{\oplus n}$. The assumption that $\gamma^{-1}\left(\operatorname{Fitt}^{0}\left(\Omega_{X / Y}\right)\right)=\left(t^{e}\right)$ implies that in suitable bases we may write $A=\left(\operatorname{diag}\left(t^{a_{1}}, \ldots, t^{a_{n}}\right), \mathbf{0}_{s, n}\right)$, for some $a_{1}, \ldots, a_{n} \geq 0$ with $\sum_{i} a_{i}=e$. We have identified the linear map $T_{\gamma}\left(f_{\infty}\right)$ with

$$
\operatorname{Hom}(A, k \llbracket t \rrbracket): \operatorname{Hom}\left(\Omega_{X, x} \otimes k \llbracket t \rrbracket, k \llbracket t \rrbracket\right) \simeq k \llbracket t \rrbracket^{\oplus n} \rightarrow \operatorname{Hom}\left(\Omega_{Y, y} \otimes k \llbracket t \rrbracket, k \llbracket t \rrbracket\right) \simeq k \llbracket t \rrbracket^{\oplus n}
$$

that is given by $\operatorname{diag}\left(t^{a_{1}}, \ldots, t^{a_{n}}\right)$. Therefore $T_{\gamma}\left(f_{\infty}\right)$ is injective, and its cokernel has dimension $e$.

\section{Generically finite morphisms Between smooth Varieties}

Suppose that $V$ is a vector space over $k$ (typically of infinite dimension). We denote by $\widehat{\operatorname{Sym}(V)}$ the completion of the symmetric algebra $\operatorname{Sym}(V)$ at the maximal ideal $\oplus_{i>0} \operatorname{Sym}^{i}(V)$. This is a local ring, and if $\mathfrak{m}$ denotes its maximal ideal, then $\widehat{\operatorname{Sym}(V)}) / \mathfrak{m}=k$ and $\mathfrak{m} / \mathfrak{m}^{2} \simeq V$.

Lemma 3.1. If $X$ is a smooth variety over $k$ and if $\gamma$ is an arc on $X$, then there is a vector space $V$ such that

$$
\widehat{\mathcal{O}_{J_{\infty}(X), \gamma}} \simeq \widehat{\operatorname{Sym}(V)}
$$

Proof. If $\operatorname{dim}(X)=0$, then we may take $V=0$, hence we now assume that $n=$ $\operatorname{dim}(X)>0$. Suppose that $\gamma$ lies over $x \in X$. After replacing $X$ by an open neighborhood of $x$, we may assume that $X=\operatorname{Spec} R$ is affine and that we have an étale map $h: X \rightarrow \mathbf{A}^{n}$ with $h(x)=0$, that corresponds to $S=k\left[y_{1}, \ldots, y_{n}\right] \rightarrow R$. We have a Cartesian diagram

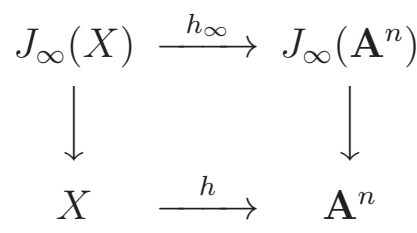

(see, for example, Lemma 2.9 in $[\mathrm{EM}])$. Since $J_{\infty}\left(\mathbf{A}^{n}\right) \simeq \operatorname{Spec} S\left[x_{i} \mid i \geq 1\right]$, we conclude that $J_{\infty}(X) \simeq \operatorname{Spec} R\left[x_{i} \mid i \geq 1\right]$, and Lemma 3.2 below implies that the formal neighborhoods of $\gamma$ and $h_{\infty}(\gamma)$ are isomorphic. Moreover, there is an automorphism 
of $J_{\infty}\left(\mathbf{A}^{n}\right)$ that takes $h_{\infty}(\gamma)$ to the constant arc $\gamma_{0}$ over the origin. As we have mentioned already, $J_{\infty}\left(\mathbf{A}^{n}\right)$ is affine with coordinate ring isomorphic to $\operatorname{Sym}\left(k^{(\mathbf{N})}\right)$, and such that the ideal of $\gamma_{0}$ corresponds to $\oplus_{i>0} \operatorname{Sym}^{i}\left(k^{(\mathbf{N})}\right)$. This gives the conclusion of the lemma.

Lemma 3.2. Let $\varphi: S \rightarrow R$ be an étale morphism of $k$-algebras of finite type, and let $\psi: S_{\infty}:=S\left[x_{i} \mid i \geq 1\right] \rightarrow R_{\infty}:=R\left[x_{i} \mid i \geq 1\right]$ be the induced map between the corresponding polynomial rings in countably many variables. If $\mathfrak{m}$ is a maximal ideal in $R_{\infty}$ with residue field $k$, and if $\mathfrak{n}=\psi^{-1}(\mathfrak{m})$, then we get an induced ring isomorphism $\widehat{\left(S_{\infty}\right)_{\mathfrak{n}}} \simeq \widehat{\left(R_{\infty}\right)_{\mathfrak{m}}}$

Proof. It is enough to show that for every local $k$-algebra $\left(T, \mathfrak{m}_{T}\right)$, with residue field $k$, and such that $\mathfrak{m}_{T}^{r}=(0)$ for some $r \geq 1$, the induced map

$$
\operatorname{Hom}_{\mathrm{loc}}\left(\left(R_{\infty}\right)_{\mathfrak{m}}, T\right) \rightarrow \operatorname{Hom}_{\mathrm{loc}}\left(\left(S_{\infty}\right)_{\mathfrak{n}}, T\right)
$$

is a bijection (here we denote by Hom loc the set of local morphisms of local $k$-algebras).

Let us denote by $\mathfrak{m}_{0}$ and $\mathfrak{n}_{0}$ the intersections of $\mathfrak{m}$ and $\mathfrak{n}$ with $R$ and $S$, respectively. By assumption, for every $i$ we have some $a_{i} \in k$ such that $x_{i}-a_{i} \in \mathfrak{m}$ (hence also $x_{i}-a_{i} \in$ $\mathfrak{n})$. It is straightforward to check that giving a local homomorphism $g:\left(S_{\infty}\right)_{\mathfrak{n}} \rightarrow T$ is equivalent to giving a local homomorphism $S_{\mathfrak{n}_{0}} \rightarrow T$, and elements $u_{i} \in T$ such that $u_{i}-a_{i} \in \mathfrak{m}_{T}$ for every $i$ (more precisely, $g$ maps each $x_{i}$ to $u_{i}$ ). A similar description holds for the local homomorphisms $\left(R_{\infty}\right)_{\mathfrak{m}} \rightarrow T$. On the other hand, since $\varphi$ is étale, the induced map

$$
\operatorname{Hom}_{\text {loc }}\left(R_{\mathfrak{m}_{0}}, T\right) \rightarrow \operatorname{Hom}_{\text {loc }}\left(S_{\mathfrak{n}_{0}}, T\right)
$$

is bijective (see, for example, $\S 28$ in [Mat] for the basics on étale ring morphisms). Putting together the above facts, we conclude that (1) is a bijection.

Proof of Theorem 1.1. Let $\left(A, \mathfrak{m}_{A}\right)$ and $\left(B, \mathfrak{m}_{B}\right)$ denote the local rings corresponding to the formal neighborhoods of $\gamma$ and $\delta$, respectively, and let $u: B \rightarrow A$ be the local homomorphism induced by $f_{\infty}$. It follows from Theorem 1.2 that if we denote by $\bar{u}$ the induced $k$-linear map $\mathfrak{m}_{B} / \mathfrak{m}_{B}^{2} \rightarrow \mathfrak{m}_{A} / \mathfrak{m}_{A}^{2}$, then $\bar{u}^{*}=\operatorname{Hom}_{k}(\bar{u}, k)$ is injective and $\operatorname{dim}_{k} \operatorname{coker}\left(\bar{u}^{*}\right)=e$. Therefore $\bar{u}$ is surjective and $\operatorname{dim}_{k} \operatorname{ker}(\bar{u})=e$.

Let $U$ be a complement of $V:=\operatorname{ker}(\bar{u})$ in $\mathfrak{m}_{B} / \mathfrak{m}_{B}^{2}$. It follows from Lemma 3.1 that we have an isomorphism $B \simeq \widehat{\operatorname{Sym}(U)} \widehat{\otimes} \widehat{\operatorname{Sym}(V)}$. On the other hand, the map $v$ given by the composition $\widehat{\operatorname{Sym}(U)} \rightarrow B \rightarrow A$ induces an isomorphism $\bar{v}: U \rightarrow \mathfrak{m}_{A} / \mathfrak{m}_{A}^{2}$. Since $\widehat{\operatorname{Sym}(U)}$ is complete, it follows that $v$ is surjective. Moreover, by Lemma 3.1 we have $A \simeq \operatorname{Sym} \widehat{\left(\mathfrak{m}_{A} / \mathfrak{m}_{A}^{2}\right)}$, hence we can find a local homomorphism $w: A \rightarrow \widehat{\operatorname{Sym}(U)}$ 
that induces $\bar{w}=\bar{v}^{-1}: \mathfrak{m}_{A} / \mathfrak{m}_{A}^{2} \rightarrow U$, and such that $v \circ w=1_{A}$. We deduce that $w$ is surjective, hence an isomorphism, and therefore $v$ is an isomorphism as well.

\section{REFERENCES}

[deJ] A. J. de Jong, Smoothness, semi-stability and alterations, Inst. Hautes Études Sci. Publ. Math. No. 83 (1996), 51-93. 3

[DL] J. Denef and F. Loeser, Germs of arcs on singular algebraic varieties and motivic integration, Invent. Math. 135 (1999), 201-232. 2, 3

[Dr] V. Drinfeld, On the Grinberg - Kazhdan formal arc theorem, preprint available at math.AG/0203263. 2

[EM] L. Ein and M. Mustaţă, Jet schemes and singularities, preprint available at arxiv: math/AG.0612862. 1, 3, 4

[Eis] D. Eisenbud, Commutative algebra. With a view toward algebraic geometry, Graduate Texts in Mathematics 150, Springer-Verlag, New York, 1995. 3

[GK] M. Grinberg, and D. Kazhdan, Versal deformations of formal arcs, Geom. Funct. Anal. 10 (2000), 543-555. 2

[Kon] M. Kontsevich, Lecture at Orsay (December 7, 1995). 2

[Lo] E. Looijenga, Motivic measures, in Séminaire Bourbaki, Vol. 1999/2000, Astérisque 276 (2002), 267-297. 3

[Mat] H. Matsumura, Commutative ring theory, Cambridge Studies in Advanced Mathematics 8, Cambridge University Press, Cambridge, 1989. 5

Department of Mathematics, University of Illinois at Chicago, 851 South MorGan Street (M/C 249), Chicago, IL 60607-7045, USA

E-mail address: ein@math.uic.edu

Department of Mathematics, University of Michigan, Ann Arbor, Mi 48109, USA

E-mail address: mmustata@umich.edu 